\title{
Determination of Liquid Density
}

\author{
Lane C. Sander and Lorna T. Sniegoski \\ National Institute of Standards and Technology, \\ Gaithersburg, MD 20899, USA \\ lane.sander@nist.gov \\ lorna.sniegoski@nist.gov
}

Video DOI: http://doi.org/10.18434/T4DK5T

Key words: Lang Levy pipette; micropipette; oscillating tube instrumentation; pycnometer; solution density.

Accepted: December 1, 2016

Published: January 12, 2017

https://doi.org/10.6028/jres.122.008

\section{Summary}

The determination of solution density is required for conversion of liquid solution levels from a mass fraction basis to a volume fraction basis. This presentation will provide information about three approaches in common use for the determination of densities for liquids: (1) micropipette method; (2) oscillating tube instrumentation; and (3) pycnometer. The video includes a brief overview of each approach, with a laboratory demonstration of the micropipette and oscillating tube methods. ${ }^{1}$

\footnotetext{
${ }^{1}$ Contribution of the National Institute of Standards and Technology. Not subject to copyright. Certain commercial equipment, instruments, or materials are identified to specify adequately the experimental procedure. Such identification does not imply recommendation or endorsement by the National Institute of Standards and Technology, nor does it imply that the materials or equipment identified are the best available for the purpose.
} 